\title{
Morphology of sulphur-terminated compound deposits condensed on different substrates in vacuum
}

\author{
K.P. Grytsenko ${ }^{1}$, P.M. Lytvyn ${ }^{1}$, T.P. Doroshenko ${ }^{1}$, Yu.V. Kolomzarov ${ }^{1}$, \\ J.L. Bricks ${ }^{2}$, V.V. Kurdyukov ${ }^{2}$,Yu.L. Slominskii ${ }^{2}$, O.I. Tolmachev ${ }^{2}$ \\ ${ }^{1}$ V. Lashkaryov Institute of Semiconductor Physics, 41, prospect Nauky, 03680 Kyiv, Ukraine, \\ ${ }^{2}$ Institute of Organic Chemistry, NAS of Ukraine, 5, Murmanska str., 02660 Kyiv, Ukraine, \\ *Corresponding author: +38(044) 5255530; e-mail:d.grytsenko@gmail.com
}

\begin{abstract}
Thin films of new sulphur-terminated organic compounds were deposited by evaporation in vacuum onto the glass, silicone, gold and polytetrafluoroethylene substrates. The influence of compound chemical structure and substrate type on morphology of the condensed solid was studied using atomic force microscopy. The significant difference in morphology of the same dye but deposited on various substrates was found.
\end{abstract}

Keywords: dye, gold, polytetrafluoroethylene, film, nanowire, self-assembly.

Manuscript received 12.05.15; revised version received 06.08.15; accepted for publication 28.10.15; published online 03.12.15.

\section{Introduction}

The design of optic and electronic chips with nano-sized active units needs methods for creation of such nanosized domains in the multilayered systems of different materials. The self-assembly method seems perspective especially in the field of fast growing organic electronics. But for self-assembly, organic compounds with special properties are necessary, taking into account not only molecule active function, but also different sticking probability on the various materials of the chip. In some cases, the ability of the molecules to form organized domains is necessary, while in other cases, when the smooth uniform film is required, this ability have to be avoided. The understanding of the influential factors to control deposit morphology to produce smooth thin film or nano-sized islands and nanowires with predetermined optical properties have been recognized as important scientific knowledge [1-4]. In the systems with gold electrodes, STOC are used for creation of self-assembled functional deposits [5]. Wet methods are mainly used for deposits manufactoring. Production of the oriented organic films by deposition in vacuum using hydrogen bonding between molecules was reported in [6]. The same STOC were used there. But no systematic studies of deposit morphology for STOC evapored on solid surfaces were found out.

The aim of this research is to study the influence of the substrate material and sulphur-terminated organic molecule chemical structure on morphology of the solids deposited by evaporation in vacuum.

\section{Experimental details}

The structures of the series of conjugated STOC, bearing thiocarbonyl terminal groups are presented in Fig. 1. Their general formula is as follows: donor-( $\pi$-system)acceptor-sulphur.

The types of STOC include: 1 - hemisquaraine 3butoxy-4-thioxo-2-((1,3,3-trimethylindolin-2-ylidene) methyl) cyclobut-2-enone (HSq); 2 - nullmethine merocyanines derivative of dihydropyridine containing thio- 
barbituric acid residue 5-(2,6-dimethyl-1-phenylpyridin-4(1H)-ylidene)-1,3-diethyl-2-thioxodihydropyrimidine-4,6(1H,5H)-dione (NMMS); 3 - nullmethine merocyanines derivative of pyrane containing thiobarbituric acid residue 5-(2,6-dimethyl-4H-pyran-4-ylidene)-1,3diphenyl-2-thioxodihydropyrimidine-4,6(1H,5H)-dione (NMMS2); 4 - 2-butyl-1,3-dimethylcyclohepta[c]pyrrole-6(2H)-thione (BDCPT). 5 - 5-[4-(dimethylamino) benzylidene]-2-thioxo-1,3-thiazolidin-4-one (DBTT). 6 - 2-thioxo-5-[2-(1,4,4-trimethyl-3,4-dihydro-2(1H)quinolinylidene)ethylidene]-1,3-thiazoli-din-4-one 5(2,3,6,7-tetrahydro-1 $H, 5 H$-pyrido[3,2,1-ij]quino-lin-9ylmethylene)-2-thioxo-1,3-thiazolidin-4-one (TTDQE). 5-\{[5-(diethylamino)-2-thienyl $]$ methylene $\}$-2-thioxo1,3-thiazolidin-4-one (DTMTT).

Synthesis of HSq was described in [7]. NMMS have been synthesized using conventional organic chemistry synthetic approaches: NMMS2 - by condensation of 2,6-dimethyl- $\gamma$-pyrone with substituted thiobarbituric acid; NMMS - by the aniline treatment of dye analogue of NMMS2 [8]. Preparation of BDCPT was described in [9]. Merocyanines DBTT, TTDQE and DTMTT are the rhodanine derivatives. They were prepared by condensation of the corresponding aldehydes with 2-thioxothiazolidin-4-one under standard conditions - by heating of reagents in polar solvent in the presence of organic base [10]. The structures of all dyes were confirmed by ${ }^{1} \mathrm{H}$ NMR spectra and elemental analysis.
The infrared spectra (IRS) of STOC were measured using Bruker Vertex 70 FTIR-spectrometer. For IRS measurements, the STOC powder was mixed with $\mathrm{KBr}$ powder and pressed in a disc.

Films were deposited using UVN-74 and VUP-5 (made in Ukraine) vacuum installations. Starting pressure in the chambers was $10^{-3} \mathrm{~Pa}$. Optical spectra of the growing films on rotating glass disc were recorded in situ. Deposition was finished when the film transmission reach nearby $50 \%$ at the absorption band maximum on glass substrate. The films were deposited on $\mathrm{Au}$, glass and polytetrafluoroethylene (PTFE) surfaces simultaneously in one run. PTFE films were deposited by decomposition of the bulk PTFE pellets and activation of the gasses with cloud of accelerated electrons. STOC and $\mathrm{Au}$ were evaporated from thermally heated crucibles. Details of the deposition processes can be found elsewhere $[11,12]$. Film morphology and thickness were studied with atomic force microscope (AFM) Nanoscope IIIa Dimension $3000^{\mathrm{TM}}$ at room temperature in the tapping mode.

\section{Results and discussion}

In IRS of STOC, the $\mathrm{C}=\mathrm{S}$ stretching frequencies have been compared with the corresponding frequencies for 1,3-diethylthiobarbituric acid.

Table. The STOC $\mathrm{C}=\mathrm{S}$ stretching frequencies of infrared spectra.<smiles>CN1C(=Cc2c([Hg][SiH3])c(=S)c2=O)C(C)(C)c2ccccc21</smiles><smiles>CCCCn1c(C)c2ccc(=S)ccc2c1C</smiles>

BDCPT<smiles>O=C1NC(=S)S/C1=C\c1cc2c3c(c1)CCCN3CCC2</smiles>

DTMTT<smiles>CCCCCCN1C(C)=CC(=C2C(=O)N(C)C(=S)N(C)C2=O)C=C1C</smiles>
NMMS<smiles>CCc1ccc(C=C2SC(=S)NC2=O)cc1</smiles>

DBTT

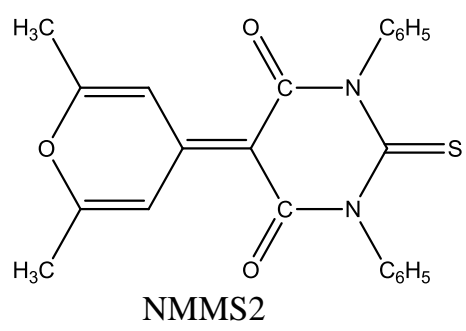<smiles>NCc1ccc(/C=C2\SC(=S)NC2=O)s1</smiles>

TTDQE

Fig. 1. Structures of the sulphur-terminated evaporable organic compounds. 


\begin{tabular}{|c|c|}
\hline Compound & $v_{\mathrm{C}=\mathrm{S}}, \mathrm{cm}^{-1}$ \\
\hline $\mathrm{HSq}$ & $1523,1269,1060$ \\
\hline NMMS & $1477,1365,1104$ \\
\hline NMMS2 & $1468,1353,973$ \\
\hline BDCPT & $1581,1384,1070$ \\
\hline 1,3-diethylthiobarbituric acid & $1434,1257,1106$ \\
\hline
\end{tabular}

Comparison of the measured values of frequencies, presented in the Table, revealed that the thiocarbonyl group in all STOC is polarized like that in the 1,3diethylthiobarbituric acid. Thus, it is possible to assume that these compounds could interact with Au surface.

The AFM images of the Au, glass and PTFE surfaces with condensed deposits of different STOC are presented in Figs. 2-6. Several STOC produced deposits

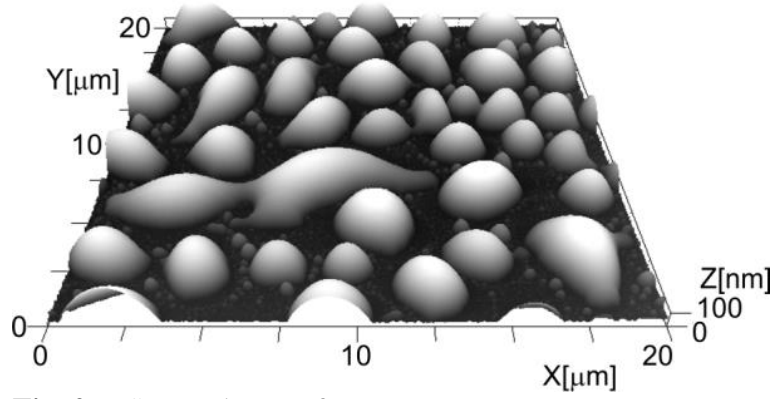

of island type on all the substrates, for example, $\mathrm{HSq}$ (Fig. 2). Some STOC produced almost the same morphology on all the substrates used. Many other STOC produced the most smooth film on Au substrate, while on PTFE and glass they produced drops, bricks and the like large units [13]. The island deposit, formed on glass substrate, should be more uniform than that formed on PTFE surface, taking into account that the surface energy of PTFE is lower than the surface energy of glass. But in our experiments, the aggregates with largest size were formed on glass surface and the smooth films were formed on $\mathrm{Au}$ surface with DBTT, NMMS, NMMS2 and BDCPT STOC (Figs. 3 and 4). In some rare cases, the smallest aggregates were formed on PTFE surface, while the larger islands were formed on the $\mathrm{Au}$ surface, for example, with DTMTT and TTDQE (Figs. 5 and 6). The general rule for smooth film is that interaction of dye molecule with substrate surface should be stronger than intermolecular interaction, while for aggregates formation the rule is vice versa. But it is true only at the first surface layer of the STOC. The next layers are free of the substrate influence. The structure of these layers is determined by order ignited by first surface STOC layer and equilibrium structure of STOC itself.

Fig. 2. HSq on glass surface.
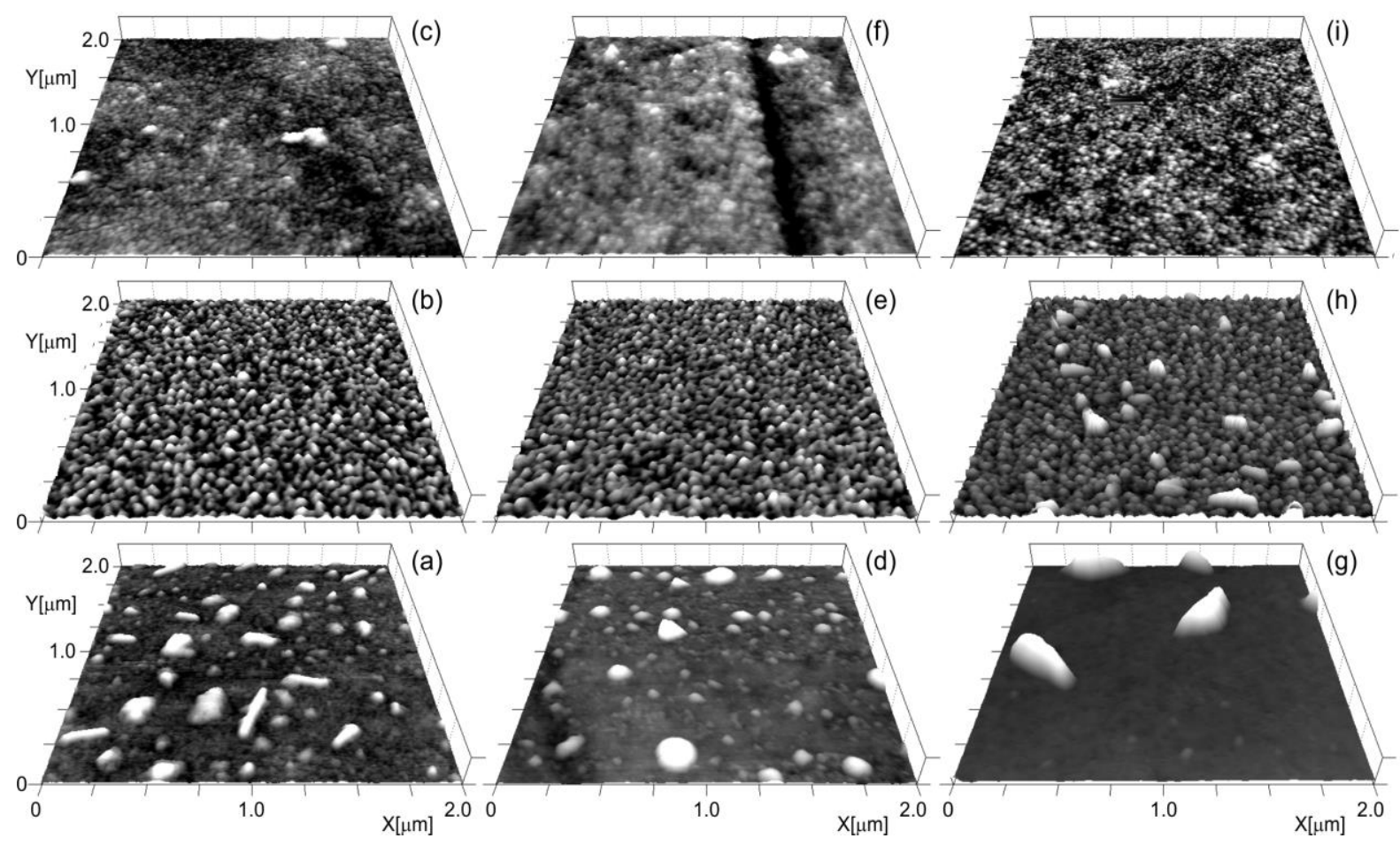

Fig. 3. DBTT on glass (a), PTFE (b), Au (c); NMMS on glass (d), PTFE (e), Au (f); NMM2 on glass (g), PTFE (h), Au (i). 


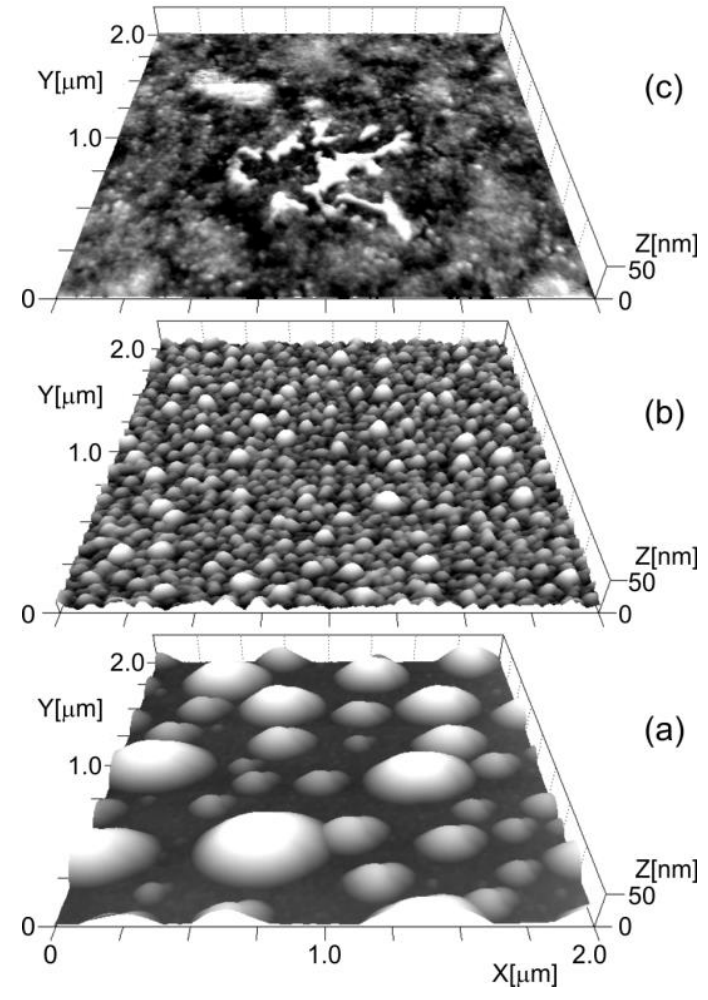

Fig. 4. BDCPT on glass, PTFE and $\mathrm{Au}$ (a, b, c, correspondingly).

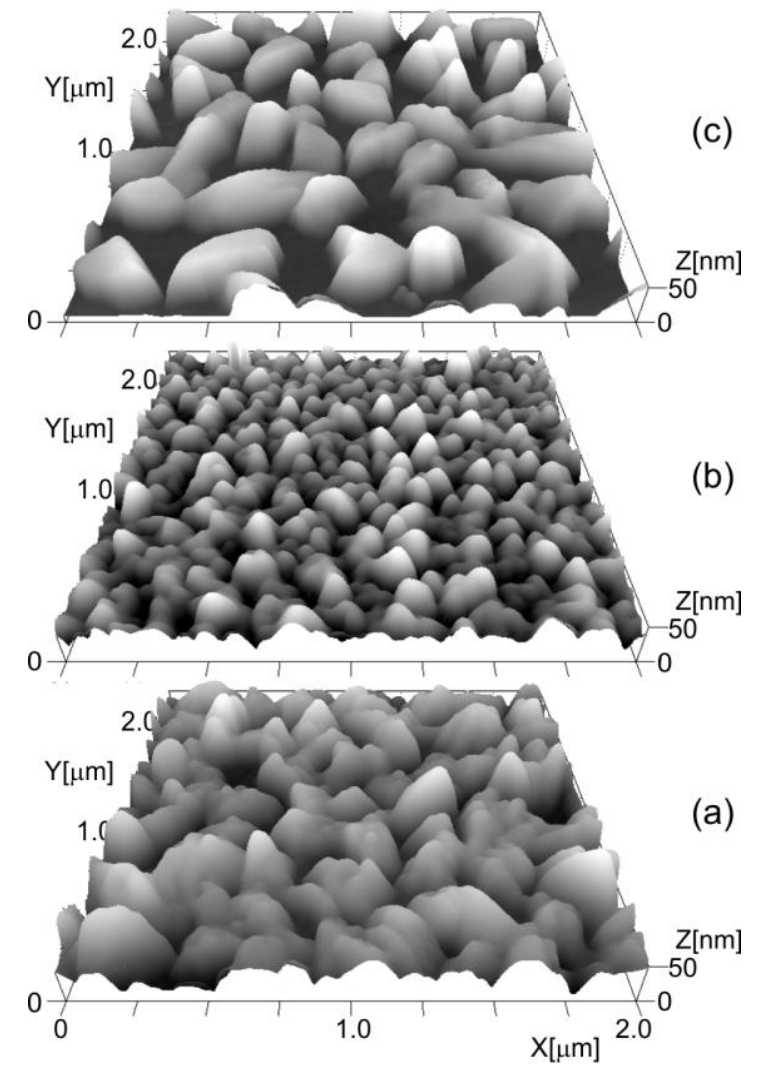

Fig. 5. DTMTT on glass (a), PTFE (b), Au (c).

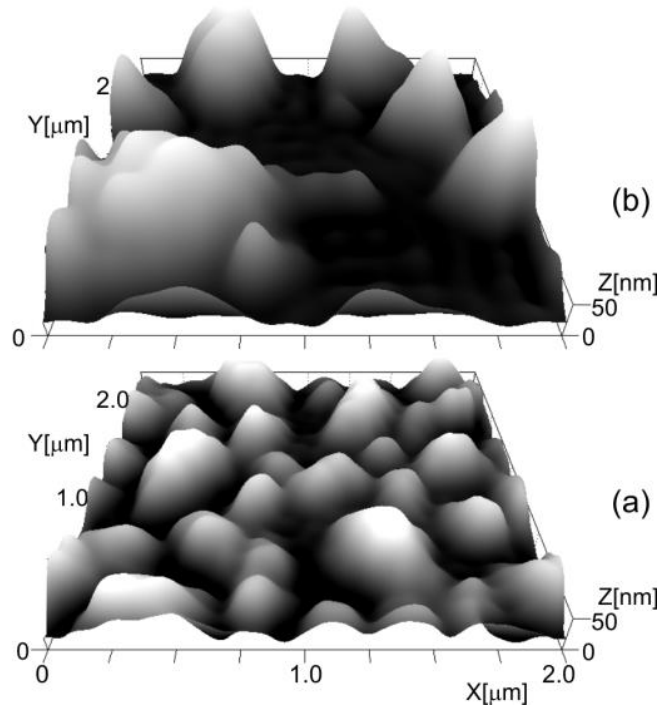

Fig. 6. TTDQE on PTFE (a), Au (b).

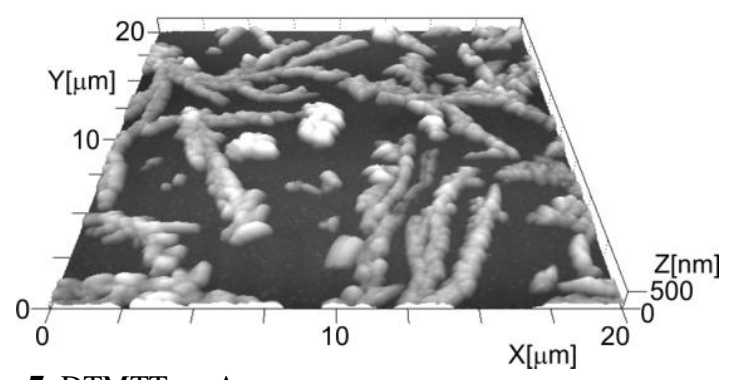

Fig. 7. DTMTT on Au.

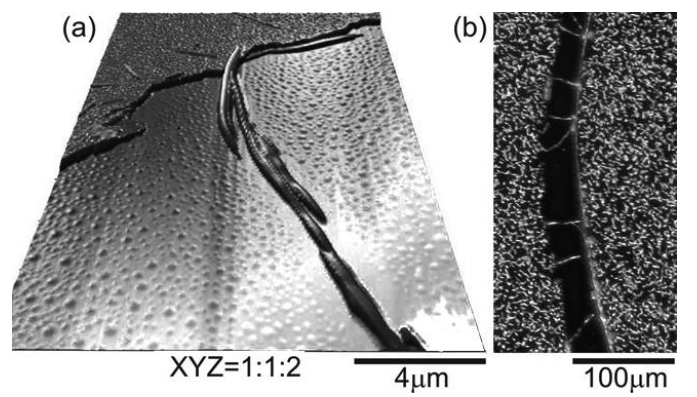

Fig. 8. Organic nanowires on $\mathrm{Si}$ surface between two gold strips.

The DTMTT formed elongated aggregates like dendrites on the Au surface, depending on deposition technology (Fig. 7). The several STOC with a most different organization on glass, $\mathrm{Au}$ and PTFE with inclination to form elongated aggregates were chosen for further researches of self-assembly in multi-layered systems. The same compounds with new chemical structure formed nanowires between gold strips on silicone and on PTFE surfaces (Fig. 8). Here, formation of nanowire lying on silicone surface but with both ends attached to gold surfaces was influenced by interplay of interactions with three materials of the system. First, it is the nanowire, grown between two gold strips due to self-

(C) 2015, V. Lashkaryov Institute of Semiconductor Physics, National Academy of Sciences of Ukraine 
assembly. In researches made before, the second end of nanowire is loose $[14,15]$. The additional efforts are necessary to connect loose end with another material surface.

\section{Conclusions}

Many evaporable sulphur-terminated organic compounds have been found out. The compounds form deposits with various morphology on different substrates. By modification of chromophore groups with various ends and side groups, it is possible to control deposit morphology, thus to make possible the selfassembly of the nano-sized organized units or, vice versa, to form the smooth and uniform film. Selfassembled organic nanowires have been obtained on the silicone surface between two gold strips.

\section{References}

1. R. Nitsche, Optical properties of organic semiconductors: from sub-monolayers to crystalline films. Diss. Dr. rer. nat., Dresden, 2005, p. 173.

2. P. Graziosi, Materials engineering in hybrid spintronic devices. Diss. Dr. rer. nat., Parma, 2010, p. 124.

3. S.A. Burke, J.M. Topple and P. Grutter, Molecular dewetting on insulators // J. Phys.: Condensed Matter, 21, p. 1-16 (2009).

4. J.M. Topple, S.A. Burke, W. Li, S. Fostner, A Tekiel, and P. Grutter, Tailoring the morphology and dewetting of an organic thin film // J. Phys. Chem. C, 115, p. 217-224 (2012).

5. A. Saywell, G. Magnano, C.J. Satterley et al., Selfassembled aggregates formed by single-molecule magnets on a gold surface // Nature Communs. 1 , Article number: 75 (2010).

6. D. Frattarelli, M. Schiavo, A. Facchetti, M.A. Ratner, and T.J. Marks, Self-assembly from the gasphase: Design and implementation of smallmolecule chromophore precursors with large nonlinear optical responses // J. Amer. Chem. Soc. 131(35), p. 12595-12612 (2009).
7. S.V. Shishkina, V.I. Baulter, O.V. Shishkin, A.L. Tatarets, L.D. Patsenker, Molecular and crystal structure of 3-butoxy-4-(1,3,3-trimethyl-2,3dihydro-1H-2-indolylidenemethyl)-3-cyclobutene1,2-dione and its thio analog // Zhurnal Struct. Khimii, 46(1), p. 156-160 (2005).

8. O.P. Dimitriev, K.P. Grytsenko, P.M. Lytvyn, et al., Substrate-induced self-assembly of donoracceptor type compounds with terminal thiocarbonyl groups // Thin Solid Films, 539, p. 127-133 (2013).

9. J. Bricks, A. Ryabitskii, A. Kachkovskii, Studies of 2-Azaazulenium Derivatives: The Natures of Electron Transitions in the 2-Azaazulenium Cation and in Monomethine Cyanine Dyes Bearing 2Azaazulenium Moieties as Terminal Groups // Eur. J. Organic Chem. 20, p. 3439-3449 (2009).

10. F.M. Hamer, The Cyanine Dyes and Related Compounds. Interscience, New York, 1964.

11. K.P. Gritsenko, A.M. Krasovsky, Thin film deposition of polymers by vacuum degradation // Chem. Rev. 103(9) p. 3607-3650 (2003).

12. K. Grytsenko, P. Lytvyn, T. Doroshenko et al., Development of new dyes for chemical binding and formation of complex nanostructures by evaporation in vacuum. EMRS Spring Meeting, May 913, 2011, p. N-19.

13. K. Grytsenko, P. Lytvyn, T. Doroshenko, O. Tolmachev, Yu. Slominskii, Yu. Briks, V. Ksianzou, S. Schrader, T. Galstian, Kinetics of dye film growth in vacuum and their selfassembled structures. Abstr. Book: Conference "Winter School on Organic Electronics", 2012, March 4-9, Planneralm, p. 73.

14. K. Xiao, I. N. Ivanov, A. A. Puretzky, Z. Liu, and D.B. Geohegan, Directed Integration of Tetracyanoquinodimethane-Cu Organic Nanowires into Prefabricated Device Architectures // Adv. Mater. 18, p. 2184-2188 (2006).

15. Y. S. Zhao, P. Zhan, J. Kim, C. Sun, and J. Huang, Patterned Growth of Vertically Aligned Organic Nanowire Waveguide Arrays // ACS NANO, 4(3), p. $1630-1636$ (2010). 